\title{
Role of diffusion-weighted imaging in evaluating therapeutic efficacy after transcatheter arterial chemoembolization for hepatocellular carcinoma
}

\author{
KEI KUBOTA, TOMOAKI YAMANISHI, SATOSHI ITOH, YORIKO MURATA, KANA MIYATAKE, \\ HIROAKI YASUNAMI, KAZUO MORIO, NORIHIKO HAMADA, AKIHITO NISHIOKA and YASUHIRO OGAWA
}

Department of Radiology, Kochi Medical School, Kochi University, Nankoku, Kochi 783-8505, Japan

Received February 25, 2010; Accepted April 30, 2010

DOI: $10.3892 /$ or_00000914

\begin{abstract}
The decision to repeat transcatheter arterial chemoembolization (TACE) for hepatocellular carcinoma (HCC) is based on correct evaluation of response to therapy. The purpose of this study was to investigate whether apparent diffusion coefficient (ADC), a quantitative parameter of diffusionweighted imaging (DWI), can predict early HCC recurrence after TACE. Results obtained using this method were compared with those using iodized-oil computed tomography (CT). DWI was performed on 25 patients with 36 HCCs before and 5-7 days after TACE to calculate the ADC of HCC. Patients were also evaluated with iodized-oil CT immediately after TACE. Contrast-enhanced CT was performed 3 months after TACE to confirm early relapse of HCC lesion. After TACE, the percent change in ADC (\%ADC) from before to after therapy was significantly increased in non-relapsed lesions $(85.2 \pm 12.4 \%)$ compared to relapsed lesions $(8.0 \pm 56.7 \%$, $\mathrm{p}=0.0004)$. However, no difference in area under the curve was seen for receiver operating characteristic analysis to predict early relapse after TACE between \%ADC from DWI (95\% confidence interval, 0.743-1.026) and iodized-oil CT (95\% confidence interval, 0.703-1.016). ADC from DWI can evaluate the efficacy of TACE for HCC as effectively as iodized-oil CT, and may help in deciding whether to repeat TACE.
\end{abstract}

\section{Introduction}

Hepatocellular carcinoma (HCC) is an aggressive tumor with a median survival following diagnosis of approximately 620 months (1). Although the mainstay of therapy is surgical resection, the majority of patients are ineligible due to tumor

Correspondence to: Dr Kei Kubota, Department of Radiology, Kochi University, Oko-cho, Nankoku-shi, Kochi 783-8505, Japan E-mail: kubotak@kochi-u.ac.jp

Key words: hepatocellular carcinoma, liver neoplasm, diffusionweighted imaging, transcatheter arterial chemoembolization, magnetic resonance imaging extension or underlying liver dysfunction. Transcatheter arterial chemoembolization (TACE) is one of the most common therapeutic options for large HCCs that are not amenable to other treatments, such as resection or radiofrequency ablation (2-4). Despite attempts at complete therapy, viable neoplastic tissue remains in some cases after TACE $(2,3)$. After treatment, follow-up imaging modalities such as computed tomography (CT), magnetic resonance imaging (MRI) and sonography have been used to assess therapeutic efficacy. However, conventional imaging necessitates injection of contrast material and shows some limitations in the evaluation of therapeutic efficacy. Since the 1990s, diffusionweighted imaging (DWI) has increasingly been used for the evaluation of extracranial sites such as the abdomen and pelvis (5-11). Effective anticancer treatment results in tumor lysis, loss of cell membrane integrity and increased extracellular space, allowing increased diffusion of water $(12,13)$. The apparent diffusion coefficient (ADC) is calculated from DWI and correlates with water diffusion without any need for injection of contrast material. Median ADC before and after TACE is significantly increased among patients with HCC $(8,9)$. Iodized-oil CT is widely used to confirm HCC response to TACE immediately after TACE (14-16). However, to the best of our knowledge, ADC has not been correlated to the degree of effectiveness of TACE against HCC as estimated by iodized-oil CT. The purpose of this study was to compare DWI and iodized-oil CT for predicting early HCC recurrence after TACE.

\section{Materials and methods}

Patients. Prospective subjects comprised 27 consecutive patients with 38 HCCs diagnosed between January and July 2009 according to characteristic imaging findings (early enhancement on dynamic contrast-enhanced CT) and positive results for serum antibody to viral hepatitis. Each patient was fully informed about the purposes and potential risks and benefits of the study and provided written consent prior to enrolment. This study was performed in accordance with the regulation of the local Ethics Committee using routine medical test. Evaluation with DWI was performed before TACE, then $36 \mathrm{HCCs}$ (mean diameter, $2.0 \mathrm{~cm}$; range, $0.8-5.2 \mathrm{~cm}$ ) in 25 patients (21 men, 4 women; mean age, 72.0 years; range, 61 92 years) depicted with DWI before TACE were re-evaluated 
A

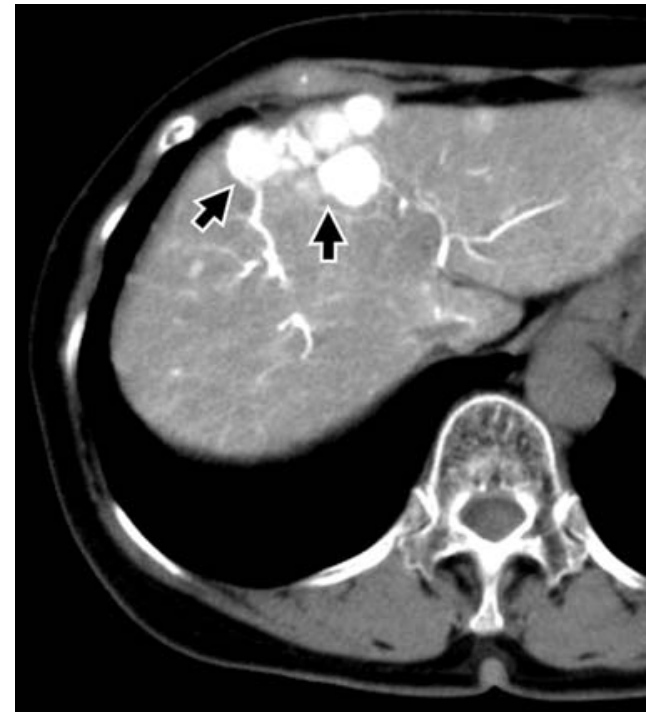

B

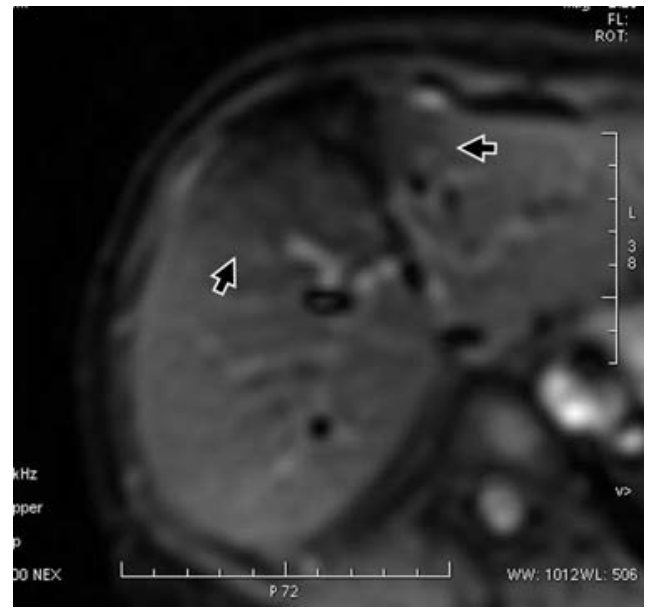

Figure 1. A 78-year-old woman with multiple HCCs in the left medial segment of the liver. (A) Contrast-enhanced CT through the intra-arterial catheter depicting multiple HCCs (arrow). (B) DWI before TACE with degraded quality due to artifact, resulting in missed tumors (arrow).

with DWI after TACE. However, two HCCs in two patients were not able to be depicted with DWI before TACE due to presumed MRI artifacts that degraded image quality (Fig. 1), and these patients were therefore excluded from the study.

MRI study. All patients were examined using a 1.5-T MRI unit (Signa HDx; GE Medical Systems, Milwaukee, WI, USA) with a $40 \mathrm{mT} / \mathrm{m}$ maximum gradient capability and built-in body coil. MRI was performed 1-2 days before and 5-7 days after TACE. DWI was obtained in 25-sec breathhold periods using a transverse spin-echo echo-planar sequence (repetition time, $4125 \mathrm{~ms}$; echo time, $56.7 \mathrm{~ms}$; gradient strength, $40 \mathrm{mT} / \mathrm{m}$; matrix size, 128x128; section thickness, $7 \mathrm{~mm}$; interslice gap, $1.5 \mathrm{~mm}$; three signals acquired; field of view, $360 \mathrm{~mm}$ ). DWI and ADC maps were acquired using bvalues of 0 and $500 \mathrm{~mm}^{2} / \mathrm{s}$ applied in the $\mathrm{z}$ direction. Quantitative ADC maps were calculated using commercially available software and an imaging workstation (Functool and AW4.3; GE Medical Systems). ADC was measured by a radiologist (K.K.) with $>20$ years of experience in hepatobiliary radiology imaging and $>10$ years of experience with DWI. Circle-shaped regions of interest (ROIs) were placed on HCCs and surrounding non-tumorous hepatic parenchyma on the monitor of the Functool workstation to calculate ADC (Fig. 2B and C; Fig. 3B and C), based on the following formula:

$$
\mathrm{ADC}=[\ln (\mathrm{s} 0 / \mathrm{s} 1)] /(\mathrm{b} 1-\mathrm{b} 0)
$$

where $\ln$ is the natural $\log , \mathrm{b} 0=0 \mathrm{~mm}^{2} / \mathrm{s}, \mathrm{b} 1=500 \mathrm{~mm}^{2} / \mathrm{s}$ and $\mathrm{s} 0$ and s1 are the signal intensities of the lesion on images obtained at each b-value. Size of the ROI was set to cover the entire tumor on the slice depicting the tumor in maximum diameter. Mean ADC of four regions of interest placed at normal hepatic parenchyma, avoiding vessel structures, was also recorded before TACE (Figs. 2B and 3B). Changes in the ADC value 5-7 days after TACE were determined by calculating the percent change in ADC from baseline (before TACE), with each patient serving as his/her own control (Fig. 2B and C; Fig. 3B and C). The percent change in ADC (\%ADC) from before to after TACE was calculated based on the following formula:

$$
\% \mathrm{ADC}=\left(\mathrm{ADC}^{\mathrm{a}}-\mathrm{ADC}^{\mathrm{b}}\right) / \mathrm{ADC}^{\mathrm{b}}
$$

where $\mathrm{ADC}^{\mathrm{b}}, \mathrm{ADC}$ of HCC before TACE; and $\mathrm{ADC}^{\mathrm{a}}, \mathrm{ADC}$ of HCC after TACE.

Iodized-oil CT. All patients underwent non-enhanced CT immediately after the TACE procedure using an angio-CT system (Asteion; Toshiba Medical Systems, Tokyo, Japan). Helical CT images were obtained in a craniocaudal direction with 5-mm-thick sections at $120 \mathrm{KVp}$ and $200 \mathrm{~mA}$ during a single breath-hold helical acquisition. CT scans were read by two radiologists (T.Y. and S.I.) with 8 and 19 years of experience, respectively, in abdominal and interventional radiological image interpretation. These investigators evaluated the success of the TACE procedure independently. Differences in assessment were resolved by consensus. As an evaluation criterion, iodized oil retention was defined as complete $(\geq 90 \%$; Fig. 2A) or incomplete (<90\%; Fig. 3A).

TACE procedure. After inserting a catheter into the appropriate hepatic artery or distal branch, TACE was performed by injecting a chemotherapeutic drug emulsion, followed by gelatin sponge particles 1-2 mm in diameter (Gelpart; NihonKayaku, Tokyo, Japan). The emulsion consisted of iodized oil (1-5 ml; Lipiodol Ultra Fluid; Terumo, Tokyo, Japan) and epirubicin hydrochloride (10-30 mg; Kyowa Hakko Kogyo, Tokyo, Japan), mixed by 10-20 passages through a three-way stopcock.

CT follow-up. For the evaluation of lesion relapse, all patients underwent follow-up CT at 3 months after TACE. Contrastenhanced dynamic CT examinations were performed using helical scanners (Aquilion; Toshiba Medical Systems). A total of $100 \mathrm{ml}$ of non-ionic contrast material (iopamidol $300 \mathrm{mgI} / \mathrm{ml}$, Bayer HealthCare, Osaka, Japan) was administered through an intravenous catheter with an automatic injector at a rate of 3-4 ml/s. Images were obtained at 45-55 and $60-70$ s after initiating injection of contrast material, representing the hepatic arterial and portal venous phases, 
A

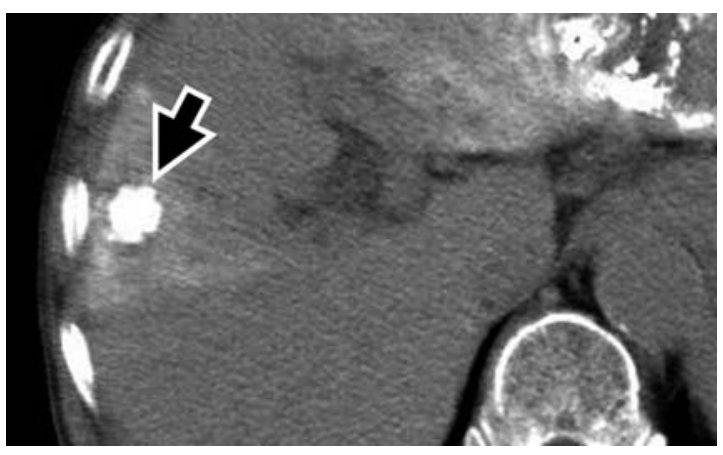

C

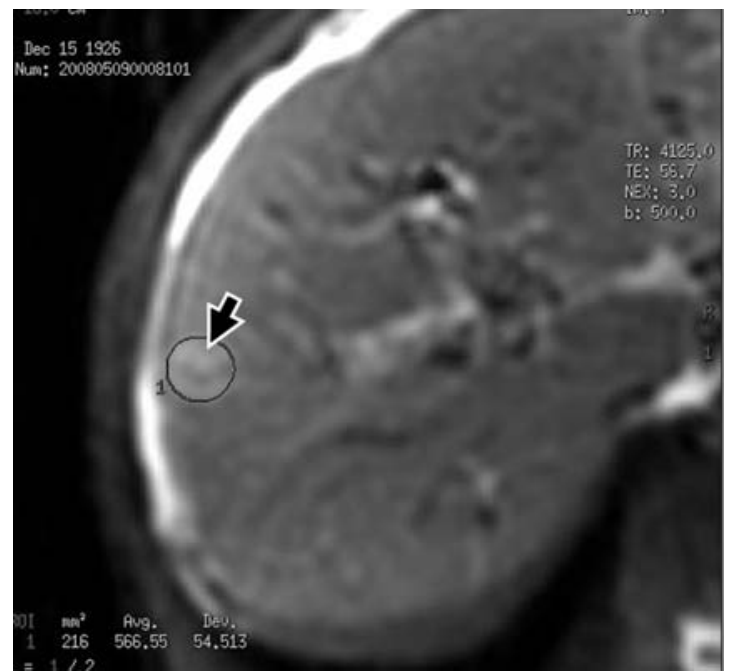

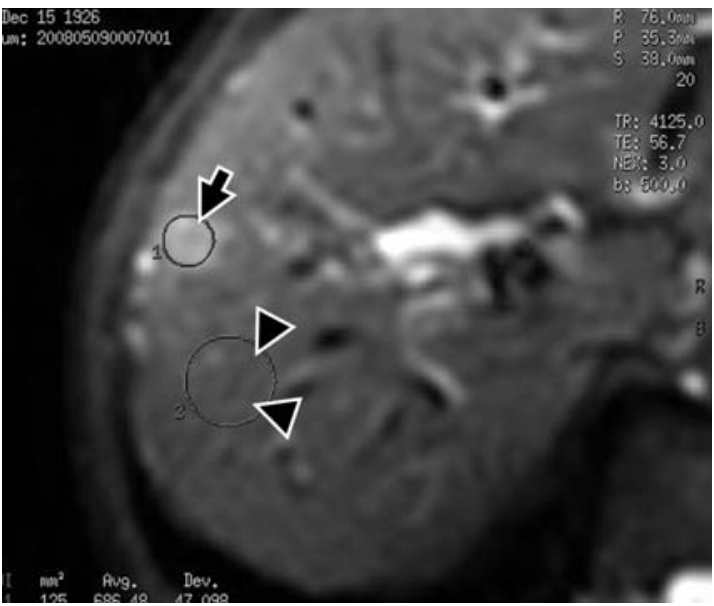

D

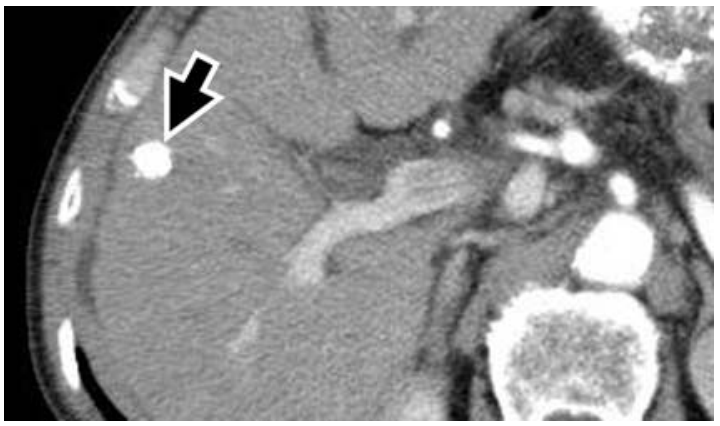

Figure 2. A 69-year-old man with a 1.1-cm HCC in the right hepatic lobe. (A) Iodized-oil CT shows complete iodized oil retention in the tumor (arrow). (B, C) DWI before (B) and after (C) TACE displayed on the Functool workstation monitor. Regions of interest were placed in the tumor (arrow) and surrounding hepatic parenchyma (arrowhead). DWI shows hyperintense signals in tumor both before and after TACE (arrow). ADC in HCC was higher after TACE $\left(3.45 \times 10^{-3} \mathrm{~mm}^{2} / \mathrm{s}\right)$ than before TACE $\left(1.15 \times 10^{-3} \mathrm{~mm}^{2} / \mathrm{s}\right)(\% \mathrm{ADC}, 200 \%)$. Mean ADC in surrounding hepatic parenchyma $\left(1.15 \times 10^{-3} \mathrm{~mm}^{2} / \mathrm{s}\right) \mathrm{resembled} \mathrm{that} \mathrm{of}$ HCC before TACE $\left(1.15 \times 10^{-3} \mathrm{~mm}^{2} / \mathrm{s}\right)$. (D) Contrast-enhanced CT at 3 months after TACE depicted only complete iodized oil retention and no relapse of tumor (arrow).

respectively. We obtained images in a craniocaudal direction at a 5-mm slice thickness and a 5-mm interval. Parameters of multi-detector CT examinations were: slice thickness, $1.0 \mathrm{~mm}$; and interval, $1.0 \mathrm{~mm}$. Tumor relapse was defined as enhancing foci near the iodized oil of an embolized lesion on follow-up CT.

Statistical analysis. Statistical analysis was performed using SPSS version 10.0 software (SPSS Inc., Chicago, IL). Mean pretreatment $\mathrm{ADC}$ values were compared between relapsed and non-relapsed lesions using the unpaired t-test. The \% ADC between relapsed and non-relapsed lesions and pretreatment ADC values between HCC lesions and surrounding hepatic parenchyma were compared using the Mann-Whitney Utest due to the unequal variance between groups. Receiver operating characteristic (ROC) analysis was performed to determine a threshold \%ADC to differentiate relapse and non-relapse of HCC lesions. Two-sided tests were used, with values of $\mathrm{p}<0.05$ indicating statistical significance.

\section{Results}

Of the 36 HCC lesions evaluated on follow-up contrastenhanced dynamic CT, 13 lesions had relapsed and 23 lesions had not. Mean ( \pm standard deviation) ADC in 36 HCCs from the 25 patients before TACE was $1.271 \pm 0.395 \times 10^{-3} \mathrm{~mm}^{2} / \mathrm{s}$, showing no significant difference from that in normal liver parenchyma $\left(1.286 \pm 0.234 \times 10^{-3} \mathrm{~mm}^{2} / \mathrm{s} ; \mathrm{p}=0.901\right.$; MannWhitney U-test). ADC before TACE did not differ significantly between relapsed $\left(1.357 \pm 0.46 \times 10^{-3} \mathrm{~mm}^{2} / \mathrm{s}\right)$ and nonrelapsed lesions $\left(1.222 \pm 0.355 \times 10^{-3} \mathrm{~mm}^{2} / \mathrm{s}\right.$, respectively; $\mathrm{p}=0.33$; unpaired t-test). Non-relapsed lesions were found to show significantly larger \%ADC than relapsed lesions $(85.2 \pm 12.4 \%$ vs. $8.0 \pm 56.7 \%$, respectively; $\mathrm{p}=0.0004$; MannWhitney U-test).

Iodized-oil CT immediately after TACE demonstrated 26 complete lesions and 10 incomplete lesions. Correlations between iodized-oil CT and relapse are shown in Table I. ROC analysis for the prediction of relapse with iodized-oil 
A

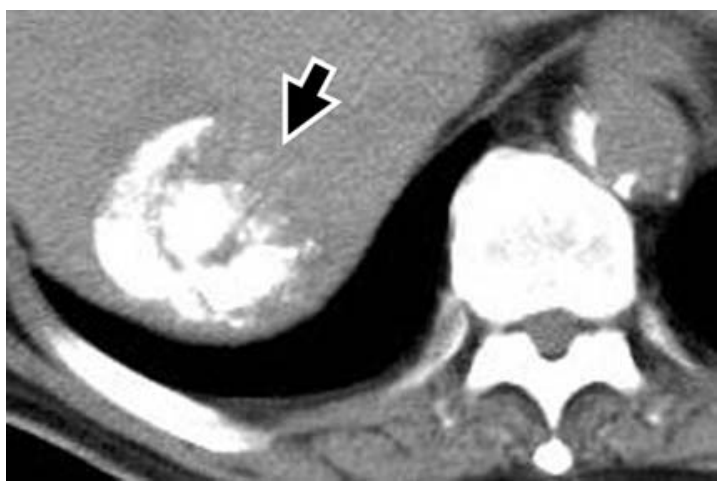

C

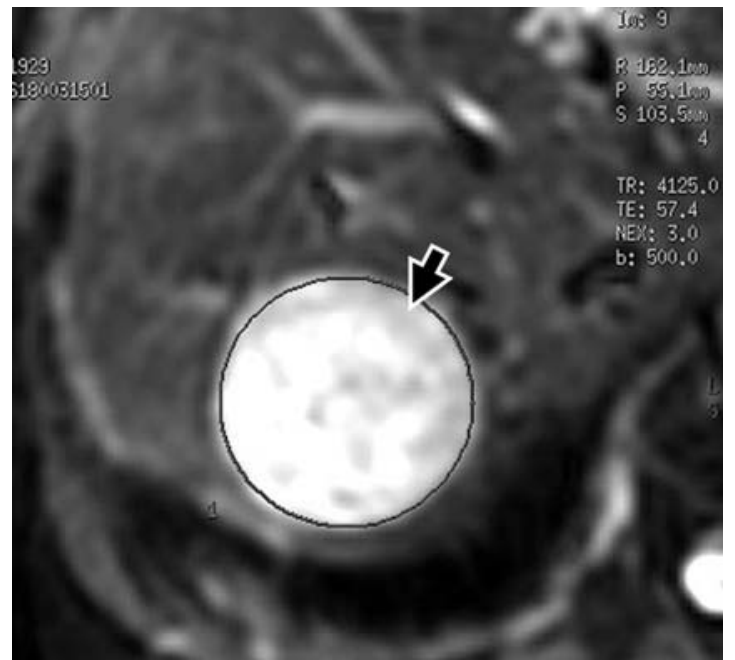

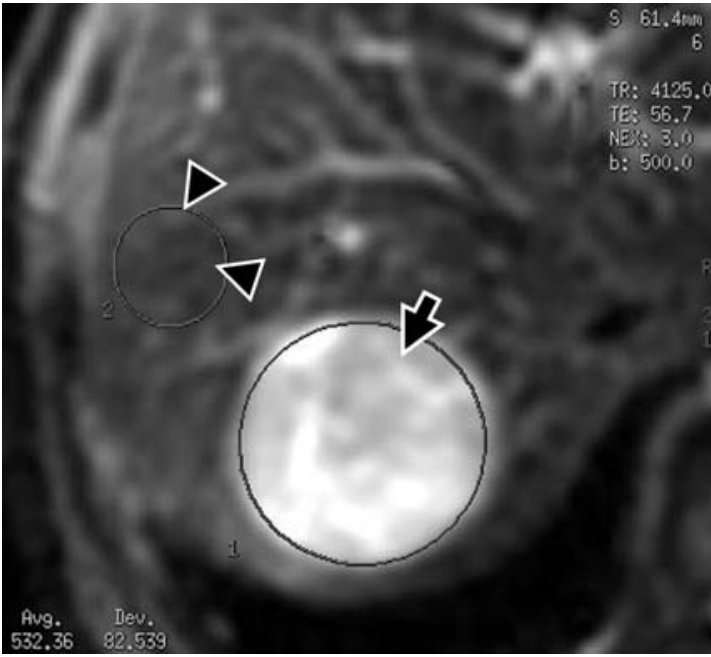

D

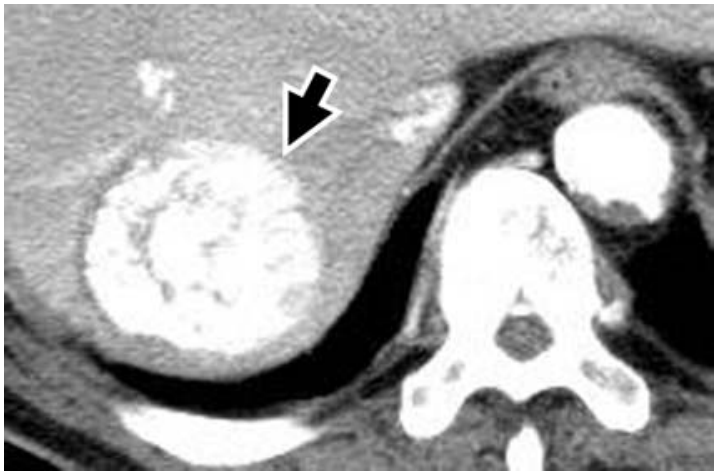

Figure 3. A 69-year-old man with a 4.8-cm HCC in the right hepatic lobe. (A) Iodized-oil CT shows incomplete retention of iodized oil in the tumor (arrow). (B, C) DWI before (B) and after (C) TACE displayed on the Functool workstation monitor. Regions of interest were placed in the tumor (arrow) and surrounding hepatic parenchyma (arrowhead). DWI shows hyperintense signals in tumor both before and after TACE (arrow). ADC in the tumor after TACE $\left(1.02 \times 10^{-3} \mathrm{~mm}^{2} / \mathrm{s}\right)$ was not elevated compared to that before TACE $\left(1.1 \times 10^{-3} \mathrm{~mm}^{2} / \mathrm{s}\right)(\%$ ADC, $-7.3 \%)$. Mean ADC in surrounding hepatic parenchyma $\left(1.21 \times 10^{-3} \mathrm{~mm}^{2} / \mathrm{s}\right)$ resembled that of HCC before TACE $\left(1.1 \times 10^{-3} \mathrm{~mm}^{2} / \mathrm{s}\right)$. (D) Contrast-enhanced CT at 3 months after TACE revealed tumor relapse close to iodized oil retention (arrow).

Table I. Correlation between iodized-oil CT findings and HCC lesion relapse.

\begin{tabular}{lrrc}
\hline & \multicolumn{3}{c}{ Relapse } \\
\cline { 2 - 4 } CT & + & - & Total \\
\hline Complete lesions & 3 & 23 & 26 \\
Incomplete lesions & 10 & 0 & 10 \\
Total & 13 & 23 & 36 \\
\hline
\end{tabular}

$\mathrm{CT}$ and \% ADC are shown in Fig. 4. The area under the curve for ROC analysis was $0.860[95 \%$ confidence interval $(\mathrm{CI})$, 0.703-1.016] for iodized-oil CT and 0.885 (95\% CI, 0.7431.026 ) for \%ADC. Sensitivity, specificity and accuracy of iodized-oil $\mathrm{CT}$ and \% ADC (threshold \% ADC value set at $13.6 \%$ ) are shown in Table II.

\section{Discussion}

HCC is a common cause of cancer death throughout the world. The majority of patients cannot undergo curative resection because of the advanced stage of the disease at the time of presentation or because of underlying cirrhosis. TACE is reportedly one of the most effective palliative measures for HCC. TACE with or without lipiodol is typically used in patients with large $\mathrm{HCC}(>3 \mathrm{~cm})$ that is not amenable to other treatments. When TACE or transcatheter arterial embolization alone was compared with conservative treatment (treatment of symptoms and complications only), survival was significantly improved with the former treatments $(4,17)$. However, despite widespread acceptance of this modality, two large randomized trials failed to demonstrate any survival advantage for TACE compared with a variety of other treatments or conservative management $(18,19)$. Although randomized controlled trials have not been performed, repeat TACE based on tumor response offers favorable prognosis compared with planned periodic TACE 


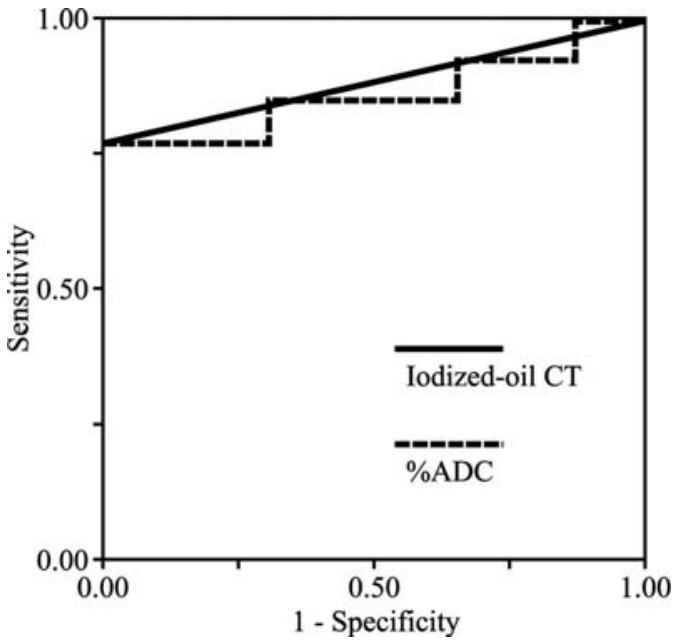

Figure 4. Results of receiver operating characteristic analyses for differentiation of relapsed and non-relapsed lesions with iodized-oil CT and $\%$ ADC.

Table II. Accuracy of iodized-oil CT findings and \%ADC in relapsed and non-relapsed lesions.

\begin{tabular}{lccc}
\hline & Sensitivity (\%) & Specificity (\%) & Accuracy (\%) \\
\hline CT & 76.9 & 100 & 91.7 \\
$\%$ ADC & 76.9 & 100 & 91.7 \\
\hline
\end{tabular}

The threshold \%ADC value was set at $13.6 \%$.

(20). TACE causes some degree of ischemic hepatic damage, which has the potential to lead to hepatic decompensation $(21,22)$. The decision to repeat TACE or add percutaneous ethanol injection therapy should be based on the assessment of residual or recurrent tumor. Accordingly, correct evaluation of tumor response and recurrence after TACE is currently one of the most interesting issues for physicians. Although patterns and distributions of iodized oil in the tumor are useful for assessing the therapeutic effects of TACE (14-16), artifacts produced by iodized oil complicate the evaluation of recurrent tumor with contrast-enhanced CT (23). Contrastenhanced MRI has been used to evaluate the efficacy of TACE $(16,24)$, but high signals on pre-contrast imaging and the contrast-enhanced fibrous capsule of the tumor also interfere with the detection of recurrent tumors $(16,25)$. Doppler or contrast-enhanced ultrasonography also shows limitations in the evaluation of HCC after TACE due to motion artifacts or ultrasound attenuation $(16,26,27)$.

DWI is a new kind of functional imaging technology that can explore the random diffusion motion of water molecules in vivo. DWI has been found to be useful for lesion detection with higher accuracy compared with superparamagnetic iron oxide-enhanced MRI (7). Quantitative DWI analyses have also been used for tumor differentiation. Malignant hepatic tumors such as metastases and HCC show higher ADC values than benign lesions such as cysts and hemangiomas $(5,6)$. Several researchers have used ADC measurement to evaluate tumor responses to treatment in cerebral gliomas (28), soft- tissue sarcoma (29) and colorectal hepatic metastases (11). Tumor lysis, loss of cell membrane integrity and an increased extracellular space are considered to contribute to increases in both water diffusion and ADC, which correlates with water diffusion $(12,13)$. The usefulness of DWI in the evaluation of therapeutic efficacy after TACE for hepatic tumors has already been reported in some animal and human studies. Some researchers have reported that areas of dead cells in VX-2 tumors in rabbits display high ADC, while areas of viable cells show low ADC after TACE $(30,31)$. ADC values also correlated with pathological percentage of necrosis after TACE in a human study (32). Kamel et al (9) and Chen et al (8) reported that the ADC value in human HCC increases after TACE. ADC was significantly higher in lesions that responded to TACE than in non-responding lesions (33). However, the percent change in ADC from baseline showed a similar area under the curve in ROC analysis for differentiation of relapsed and non-relapsed lesions with iodizedoil CT in the current study. DWI was not found to represent a reliable predictor of local HCC recurrence after TACE compared with gadolinium-enhanced MR imaging $(32,34)$. DWI also showed limitations in detecting small viable tumors after TACE in an experimental animal study (35). DWI may thus improve the prognosis of patients with HCC by allowing prompt repeat TACE based on correct early prediction of recurrent $\mathrm{HCC}$ with equivalent quality to iodized-oil $\mathrm{CT}$, but does not exceed the reliability of iodized-oil CT.

The difference in ADC values between responding and non-responding lesions before TACE produced controversial results in a study carried out by Yuan et al (33) and this study. A significantly higher pretreatment mean ADC was seen in non-responding lesions $\left(1.726 \pm 0.323 \times 10^{-3} \mathrm{~mm}^{2} / \mathrm{s}\right)$ than in responding lesions $\left(1.294 \pm 0.185 \times 10^{-3} \mathrm{~mm}^{2} / \mathrm{s}\right)$ in the study by Yuan et al (33), while no significant difference was evident in our study. Although the reason for this controversial result is unclear, all lesions showed early enhancement on dynamic contrast-enhanced CT in this study, while the presence of early enhancement is not clearly described in the study by Yuan et al (33). The response of HCC to TACE generally depends on the presence of early enhancement on contrast-enhanced $\mathrm{CT}$, which may indicate a lower ADC value in the lesion due to less necrosis (33). HCC without enhancement on contrastenhanced CT in the study by Yuan et al may thus have contributed to the discrepancy between the two studies.

DWI was not able to depict two tumors in our study population. DWI is known to have limitations such as relatively poor signal-to-noise ratio and high sensitivity to pulsatile or susceptibility artifacts, degrading the ability to detect lesions $(10,36)$.

In conclusion, ADC, a new quantitative measurement from DWI, may allow effective evaluation of the efficacy of TACE for HCC and correctly reveal recurrent tumor. However, further refinement of MRI techniques is needed to properly clarify MRI susceptibility and motion artifacts.

\section{References}

1. No authors listed: A new prognostic system for hepatocellular carcinoma: a retrospective study of 435 patients: the Cancer of the Liver Italian Program (CLIP) investigators. Hepatology 28: 751-755, 1998. 
2. Yu YQ, Xu DB, Zhou XD, Lu JZ, Tang ZY and Mack P: Experience with liver resection after hepatic arterial chemoembolization for hepatocellular carcinoma. Cancer 71: 62-65, 1993.

3. Di Carlo V, Ferrari G, Castoldi R, et al: Pre-operative chemoembolization of hepatocellular carcinoma in cirrhotic patients. Hepatogastroenterology 45: 1950-1954, 1998.

4. Llovet JM, Real MI, Montana X, et al: Arterial embolisation or chemoembolisation versus symptomatic treatment in patients with unresectable hepatocellular carcinoma: a randomised controlled trial. Lancet 359: 1734-1739, 2002.

5. Kim T, Murakami T, Takahashi S, Hori M, Tsuda K and Nakamura H: Diffusion-weighted single-shot echoplanar MR imaging for liver disease. AJR 173: 393-398, 1999.

6. Taouli B, Vilgrain V, Dumont E, Daire JL, Fan B and Menu Y: Evaluation of liver diffusion isotropy and characterization of focal hepatic lesions with two single-shot echo-planar MR imaging sequences: prospective study in 66 patients. Radiology 226: 71-78, 2003.

7. Nasu K, Kuroki Y, Nawano S, et al: Hepatic metastases: diffusion-weighted sensitivity-encoding versus SPIO-enhanced MR imaging. Radiology 239: 122-130, 2006.

8. Chen CY, Li CW, Kuo YT, et al: Early response of hepatocellular carcinoma to transcatheter arterial chemoembolization: choline levels and MR diffusion constants - initial experience. Radiology 239: 448-456, 2006.

9. Kamel IR, Bluemke DA, Eng J, et al: The role of functional MR imaging in the assessment of tumor response after chemoembolization in patients with hepatocellular carcinoma. J Vasc Interv Radiol 17: 505-512, 2006.

10. Koh DM and Collins DJ: Diffusion-weighted MRI in the body: applications and challenges in oncology. AJR 188: 1622-1635, 2007.

11. Koh DM, Scurr E, Collins D, et al: Predicting response of colorectal hepatic metastasis: value of pretreatment apparent diffusion coefficients. AJR 188: 1001-1008, 2007

12. Moffat BA, Hall DE, Stojanovska J, et al: Diffusion imaging for evaluation of tumor therapies in preclinical animal models. MAGMA 17: 249-259, 2004.

13. Moffat BA, Chenevert TL, Lawrence TS, et al: Functional diffusion map: a non-invasive MRI biomarker for early stratification of clinical brain tumor response. Proc Natl Acad Sci USA 102: 5524-5529, 2005

14. Imaeda T, Yamawaki Y, Seki M, et al: Lipiodol retention and massive necrosis after lipiodol-chemoembolization of hepatocellular carcinoma: correlation between computed tomography and histopathology. Cardiovasc Intervent Radiol 16: 209-213, 1993.

15. Takayasu K, Arii S, Matsuo N, et al: Comparison of CT findings with resected specimens after chemoembolization with iodized oil for hepatocellular carcinoma. AJR 175: 699-704, 2000.

16. Kubota K, Hisa N, Nishikawa T, et al: Evaluation of hepatocellular carcinoma after treatment with transcatheter arterial chemoembolization: comparison of Lipiodol-CT, power Doppler sonography and dynamic MRI. Abdom Imaging 26: 184-190, 2001

17. Lo CM, Ngan H, Tso WK, et al: Randomized controlled trial of transarterial lipiodol chemoembolization for unresectable hepatocellular carcinoma. Hepatology 35: 1164-1171, 2002.

18. Bruix J, Llovet JM, Castells A, et al: Transarterial embolization versus symptomatic treatment in patients with advanced hepatocellular carcinoma: results of a randomized, controlled trial in a single institution. Hepatology 27: 1578-1583, 1998.

19. Pelletier G, Ducreux M, Gay F, et al: Treatment of unresectable hepatocellular carcinoma with lipiodol chemoembolization: a multicenter randomized trial. Groupe CHC. J Hepatol 29: 129-134, 1998.
20. Ernst O, Sergent G, Mizrahi D, Delemazure O, Paris JC and L'Hermine C: Treatment of hepatocellular carcinoma by transcatheter arterial chemoembolization: comparison of planned periodic chemoembolization and chemoembolization based on tumor response. AJR 172: 59-64, 1999.

21. Chung JW, Park JH, Han JK, et al: Hepatic tumors: predisposing factors for complications of transcatheter oily chemoembolization. Radiology 198: 33-40, 1996.

22. Chan AO, Yuen MF, Hui CK, Tso WK and Lai CL: A prospective study regarding the complications of transcatheter intraarterial lipiodol chemoembolization in patients with hepatocellular carcinoma. Cancer 94: 1747-1752, 2002.

23. Kim HC, Kim AY, Han JK, et al: Hepatic arterial and portal venous phase helical CT in patients treated with transcatheter arterial chemoembolization for hepatocellular carcinoma: added value of unenhanced images. Radiology 225: 773-780, 2002.

24. Murakami T, Nakamura H, Tsuda K, et al: Treatment of hepatocellular carcinoma by chemoembolization: evaluation with 3DFT MR imaging. AJR 160: 295-299, 1993.

25. De Santis M, Alborino S, Tartoni PL, Torricelli P, Casolo A and Romagnoli R: Effects of lipiodol retention on MRI signal intensity from hepatocellular carcinoma and surrounding liver treated by chemoembolization. Eur Radiol 7: 10-16, 1997.

26. Rubin JM, Bude RO, Carson PL, Bree RL and Adler RS: Power Doppler US: a potentially useful alternative to mean frequencybased color Doppler US. Radiology 190: 853-856, 1994.

27. Kubota K, Hisa N, Fujiwara Y, Fukumoto M, Yoshida D and Yoshida S: Evaluation of the intratumoral vasculature of hepatocellular carcinoma by power Doppler sonography: advantages and disadvantages versus conventional color Doppler sonography. Abdom Imaging 25: 172-178, 2000.

28. Chenevert TL, McKeever PE and Ross BD: Monitoring early response of experimental brain tumors to therapy using diffusion magnetic resonance imaging. Clin Cancer Res 3: 1457-1466, 1997.

29. Einarsdóttir H, Karlsson M, Wejde J and Bauer HC: Diffusionweighted MRI of soft tissue tumours. Eur Radiol 14: 959-963, 2004.

30. Geschwind JF, Artemov D, Abraham S, et al: Chemoembolization of liver tumor in a rabbit model: assessment of tumor cell death with diffusion-weighted MR imaging and histologic analysis. J Vasc Interv Radiol 11: 1245-1255, 2000.

31. Yuan YH, Xiao EH, Liu JB, et al: Characteristics and pathological mechanism on magnetic resonance diffusion-weighted imaging after chemoembolization in rabbit liver VX-2 tumor model. World J Gastroenterol 13: 5699-5706, 2007.

32. Mannelli L, Kim S, Hajdu CH, Babb JS, Clark TW and Taouli B: Assessment of tumor necrosis of hepatocellular carcinoma after chemoembolization: diffusion-weighted and contrast-enhanced MRI with histopathologic correlation of the explanted liver. AJR 193: 1044-1052, 2009.

33. Yuan Z, Ye XD, Dong S, Xu LC, Xu XY, Liu SY and Xiao XS: Role of magnetic resonance diffusion-weighted imaging in evaluating response after chemoembolization of hepatocellular carcinoma. Eur J Radiol (In press).

34. Goshima S, Kanematsu M, Kondo H, Yokoyama R, Tsuge Y, Shiratori Y, Onozuka M and Moriyama N: Evaluating local hepatocellular carcinoma recurrence post-transcatheter arterial chemoembolization: is diffusion-weighted MRI reliable as an indicator? J Magn Reson Imaging 27: 834-839, 2008.

35. Youn BJ, Chung JW, Son KR, et al: Diffusion-weighted MR: therapeutic evaluation after chemoembolization of VX-2 carcinoma implanted in rabbit liver. Acad Radiol 15: 593-600, 2008.

36. Koh DM, Takahara T, Imai Y and Collins DJ: Practical aspects of assessing tumors using clinical diffusion-weighted imaging in the body. Magn Reson Med Sci 6: 211-224, 2007. 